\title{
Norms of Composition Operators on the Hardy Space
}

Matthew J. Appel, Paul S. Bourdon and John J. Thrall

\section{CONTENTS}

1. Introduction

2. Preliminaries

3. What's Known about Norms

4. Experiments and Results

References
Research supported in part by National Science Foundation grant DMS-9401206. Appel and Thrall were supported by the Foundation's REU program for work they performed as undergraduate assistants to Bourdon.
We show that the norm of a composition operator on the classical Hardy space $H^{2}$ cannot be computed using only the set of $\mathrm{H}^{2}$ reproducing kernels, answering a question raised by Cowen and MacCluer.

\section{INTRODUCTION}

Let $T$ be a bounded linear operator taking the Hilbert space $H$ into itself. Recall that the norm of $T$ is a measure of how much $T$ distorts the unit ball of $H$, and is defined as

$$
\begin{aligned}
\|T\| & =\sup \{\|T f\|: f \in H \text { with }\|f\| \leq 1\} \\
& =\sup \left\{\left\|T^{*} f\right\|: f \in H \text { with }\|f\| \leq 1\right\} .
\end{aligned}
$$

Here the norm on the right-hand side is the one induced by the inner product of $H$, and $T^{*}$ denotes the adjoint of $T$.

Calculating the exact value of the norm of a continuous Hilbert-space operator can be difficult. For example, norms of composition operators on the Hardy space $H^{2}$ have been computed only in certain special cases (described in the next section). Cowen and MacCluer [1995, p. 125] ask if the norm of a composition operator $T$ on $H^{2}$ is determined by the action of $T^{*}$ on only a small subset of the unit ball of $H^{2}$ - the set of normalized reproducing kernels for $H^{2}$.

Here we describe experiments that led to an example that answers this question in the negative. That the example fully resolves the issue raised by Cowen and MacCluer follows from Theorem 4.4, which is stated and proved in the last section of the paper. 


\section{PRELIMINARIES}

Let $H(U)$ denote the space of all functions holomorphic on the open unit disk $U=\{z:|z|<1\}$. Any function $\varphi \in H(U)$ satisfying $\varphi(U) \subset U$ induces a linear composition operator $C_{\varphi}$ on $H(U)$ defined by

$$
C_{\varphi} f=f \circ \varphi .
$$

Littlewood's Subordination Principle shows that $C_{\varphi}$ restricts to a bounded operator on the Hardy space $H^{2}$ [Littlewood 1925]. The space $H^{2}$ is the function-theoretic incarnation of the Hilbert space $\ell^{2}$ : it consists of those analytic functions on $U$ whose Taylor coefficients in the expansion about the origin are square summable.

The inner product of the Hardy space functions $f(z)=\sum_{n=0}^{\infty} a_{n} z^{n}$ and $g(z)=\sum_{n=0}^{\infty} b_{n} z^{n}$ is given by

$$
\langle f, g\rangle=\sum_{n=0}^{\infty} a_{n} \bar{b}_{n},
$$

and thus the norm of $f$ is given by

$$
\|f\|^{2}=\sum_{n=0}^{\infty}\left|a_{n}\right|^{2} .
$$

The norm of $f \in H^{2}$ is also given by

$$
\|f\|^{2}=\frac{1}{2 \pi} \int_{-\pi}^{\pi}\left|f^{*}\left(e^{i \theta}\right)\right|^{2} d \theta,
$$

where $f^{*}$ represents the radial limit function of $f$ (see [Duren 1970], for example).

The reproducing kernels for $\mathrm{H}^{2}$ will play an important role in this paper. For $\alpha \in U$, the reproducing kernel at $\alpha$, denoted $k_{\alpha}$, is defined by

$$
k_{\alpha}(z)=\frac{1}{1-\bar{\alpha} z} .
$$

The reader may verify that the label "reproducing kernel" is apt: for each $f \in H^{2}$,

$$
f(\alpha)=\left\langle f, k_{\alpha}\right\rangle .
$$

The collection of reproducing kernels for $H^{2}$ is invariant under the action of adjoint composition operators.
Proposition 2.1. Suppose that $\varphi$ maps $U$ analytically into $U$. Then $C_{\varphi}^{*}\left(k_{\alpha}\right)=k_{\varphi(\alpha)}$.

Proof. For $f \in H^{2}$ arbitrary,

$$
\left\langle f, C_{\varphi}^{*} k_{\alpha}\right\rangle=\left\langle f \circ \varphi, k_{\alpha}\right\rangle=f(\varphi(\alpha))=\left\langle f, k_{\varphi(\alpha)}\right\rangle .
$$

We are now in a position to state carefully Cowen and MacCluer's question regarding the norms of composition operators.

Question 2.2. Is there an analytic map $\varphi$ of the unit disk into itself such that, on $\mathrm{H}^{2}$,

$$
\left\|C_{\varphi}\right\|>\sup _{\alpha \in U} \frac{\left\|k_{\varphi(\alpha)}\right\|}{\left\|k_{\alpha}\right\|} ?
$$

Of course, $\left\|C_{\varphi}\right\|$ is always greater than or equal to the supremum on the right-hand side of (2.1), because

$$
\frac{\left\|k_{\varphi(\alpha)}\right\|}{\left\|k_{\alpha}\right\|}=\left\|C_{\varphi}^{*}\left(\frac{k_{\alpha}}{\left\|k_{\alpha}\right\|}\right)\right\|,
$$

and $k_{\alpha} /\left\|k_{\alpha}\right\|$ is in the unit ball of $H^{2}$ (for each $\alpha \in U)$.

We show in Section 4 that the answer to Question 2.2 is yes. Some of the results that motivated Cowen and MacCluer to raise this question are discussed in the next section.

\section{WHAT'S KNOWN ABOUT NORMS}

Throughout the remainder of this paper, $\varphi$ will denote an analytic function taking $U$ into itself, and $C_{\varphi}$ will denote the corresponding composition operator on the Hardy space $H^{2}$. A lower estimate on the norm of $C_{\varphi}$ may be obtained as follows:

$$
\left\|C_{\varphi}\right\|^{2} \geq\left\|C_{\varphi}^{*} k_{0}\right\|^{2}=\frac{1}{1-|\varphi(0)|^{2}},
$$

where the inequality holds because $k_{0}=1$ is in the unit ball of $H^{2}$. Littlewood's Subordination Principle and a change of variables argument yields the upper estimate on the norm presented in the following theorem [Ryff 1966]. 
Theorem 3.1. Suppose $\varphi$ maps $U$ analytically into itself. Then

$$
\frac{1}{1-|\varphi(0)|^{2}} \leq\left\|C_{\varphi}\right\|^{2} \leq \frac{1+|\varphi(0)|}{1-|\varphi(0)|}
$$

The preceding theorem shows that $\left\|C_{\varphi}\right\|=1$ when $\varphi(0)=0$ (this is what Littlewood proved). Because $\left\|k_{\varphi(0)}\right\|=\left\|k_{0}\right\|$ when $\varphi(0)=0$, no $\varphi$ fixing the origin will resolve Question 2.2.

The norm of the composition operator induced by a map of the form $\varphi(z)=s z+t$ was proved by Cowen [1988] to equal

$$
\left(\frac{2}{1+|s|^{2}-|t|^{2}+\sqrt{\left(1-|s|^{2}+|t|^{2}\right)^{2}-4|t|^{2}}}\right)^{\frac{1}{2}} .
$$

Cowen shows that in this case the norm equals the supremum on the right-hand side of (2.1). Certainly Cowen's formula hints at how difficult the computation of the norm of composition operators can be.

Recall that an analytic function $\varphi: U \rightarrow U$ is inner if its radial limit function has modulus one a.e. [Duren 1970]. The norms of composition operators induced by inner functions were computed by Nordgren [1968].

Theorem 3.2. Suppose $\varphi$ is inner; then

$$
\left\|C_{\varphi}\right\|^{2}=\frac{1+|\varphi(0)|}{1-|\varphi(0)|} .
$$

For univalent (one-to-one) inner functions $\varphi$, it's not difficult to show that the supremum on the right-hand side of (2.1) always yields the norm of $C_{\varphi}$. A univalent inner function must have the form

$$
z \mapsto c \frac{a-z}{1-\bar{a} z}
$$

for some $a \in U$ and $c$ of modulus 1 .

Even though no formula has been obtained yielding the norm of a composition operator on $\mathrm{H}^{2}$, there is a formula for the essential norm [Shapiro 1987]. (The essential norm of a Hilbert-space operator $T$ is the distance in the operator norm from
$T$ to the collection of compact operators.) The essential norm of $C_{\varphi}$ is not, in general, determined by the action of $C_{\varphi}^{*}$ on reproducing kernels, but is determined by such action when $\varphi$ is univalent. This fact led Shapiro to conjecture (private communication) that the answer Question 2.2 should be yes in general, perhaps no if $\varphi$ is required to be univalent. In the following section, we show that the answer is yes even for univalent $\varphi$.

For more information about the norms of composition operators, see [Cowen and MacCluer 1995], for example.

\section{EXPERIMENTS AND RESULTS}

The way to proceed in order to prove that some $\varphi$ satisfies the inequality (2.1) seems clear: try to find the supremum of values of

$$
L_{\varphi}(\alpha)=\frac{\left\|k_{\varphi(\alpha)}\right\|}{\left\|k_{\alpha}\right\|}
$$

as $\alpha$ varies over $U$, then try to show that the norm of $C_{\varphi}$ exceeds this supremum. (We've chosen the letter $L$ to denote this ratio because it will be less than or equal to the norm of $C_{\varphi}$.) The problem, then, is to guess what $\varphi$ should be.

A natural place to begin is with composition operators induced by inner functions, since their norms have been computed (Theorem 3.2). We've remarked earlier that no univalent inner function induces a composition operator satisfying (2.1). For this reason we start by considering, for example, the two-to-one inner function

$$
\psi(z)=\left(\frac{\frac{1}{2}-z}{1-\frac{1}{2} z}\right)^{2} .
$$

Figure 1, generated by Maple, shows the graph of $L_{\psi}^{2}$ over the unit disk. It suggests that the supremum of values of $L_{\psi}^{2}$ is about 1.5. On the other hand, because $\psi$ is inner, Theorem 3.2 shows that $\left\|C_{\psi}\right\|^{2}=(1+|\varphi(0)|) /(1-|\varphi(0)|)=\frac{5}{3}$. Thus, if Figure 1 is accurate, $C_{\psi}$ satisfies (2.1), answering Question 2.2 in the affirmative. 


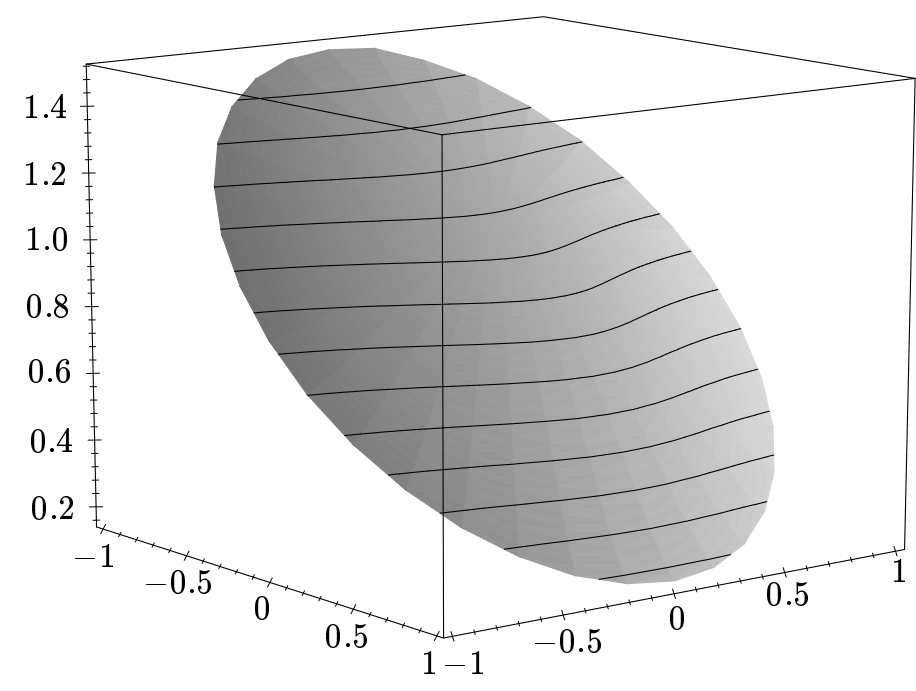

FIGURE 1. Graph of $L_{\psi}^{2}$.

Proposition 4.1. For $\psi$ as in (4.1), we have

$$
\left\|C_{\psi}\right\|>\sup _{\alpha \in U} \frac{\left\|k_{\psi(\alpha)}\right\|}{\left\|k_{\alpha}\right\|} .
$$

Proof. We need to show that $\sup _{\alpha \in U} L_{\psi}(\alpha)^{2}$ is less than $\frac{5}{3}$. Let $\alpha=r e^{i \theta}$ be the polar form of $\alpha$. Applying the definition of $L_{\psi}$, we obtain

$$
L_{\psi}(\alpha)^{2}=\frac{1-|r|^{2}}{1-\left|\frac{\frac{1}{2}-r e^{i \theta}}{1-\frac{1}{2} r e^{i \theta}}\right|^{4}} .
$$

View $r \in[0,1)$ in the preceding equation as fixed. The maximum in $\theta$ of the quantity in absolute values in the denominator on the right of (4.2) is easy to find: observe that the linear-fractional map $z \mapsto$ $\left(\frac{1}{2}-z\right) /\left(1-\frac{1}{2} z\right)$ takes the circle $\left\{r e^{i \theta}: \theta \in[0,2 \pi]\right\}$ to a circle with diameter on the real axis between the points $\left(\frac{1}{2}-r\right) /\left(1-\frac{1}{2} r\right)$ and $\left(\frac{1}{2}+r\right) /\left(1+\frac{1}{2} r\right)$; thus, the maximum of $L\left(r e^{i \theta}\right)^{2}$ is

$$
\frac{1-|r|^{2}}{1-\left|\frac{\frac{1}{2}+r}{1+\frac{1}{2} r}\right|^{4}}=\frac{(2+r)^{4}}{3\left(5+8 r+5 r^{2}\right)} .
$$

The quantity on the right increases to $\frac{3}{2}$ as $r$ increases to 1 , and thus $\sup _{\alpha \in U} L_{\psi}(\alpha)^{2}=\frac{3}{2}$, which is less than $\frac{5}{3}=\left\|C_{\psi}\right\|^{2}$.
This $C_{\psi}$, though it answers Question 2.2, is unsatisfactory in two respects: it is not univalent, and, more important, the norm of $C_{\psi}$ is determined by $C_{\psi}$ 's action on reproducing kernels! One must allow $C_{\psi}$ (instead of $C_{\psi}^{*}$ ) to act on the kernels.

Theorem 4.2. Suppose $\varphi$ is inner; then

$$
\left\|C_{\varphi}\right\|=\sup _{\alpha \in U}\left\|C_{\varphi}\left(\frac{k_{\alpha}}{\left\|k_{\alpha}\right\|}\right)\right\| .
$$

The following Lemma will facilitate the proof.

Lemma 4.3. Suppose that $f(z)=(a z+b) /(c z+d)$ is a nonconstant $H^{2}$ function; then

$$
\|f\|^{2}=\frac{|a|^{2}+|b|^{2}-2 \operatorname{Re}(\bar{a} b c / d)}{|d|^{2}-|c|^{2}} .
$$

Proof. Find the power series in $z$ for $f$; verify that it is square summable if and only if $|d|>|c|$, and that the sum of the squares of the moduli of series coefficients is given by the formula above ( $f$ is nonconstant means $a d-b c \neq 0$ ).

Proof of Theorem 4.2. We employ a fact observed by Nordgren [1968, p. 443]: if $\varphi$ is inner with $\varphi(0)=0$, then $C_{\varphi}$ is an isometry; i.e., $\left\|C_{\varphi} f\right\|=\|f\|$ for all $f \in H^{2}$. Let $\varphi$ be an arbitrary inner function and set $\beta=\varphi(0)$. The function

$$
\nu(z)=\frac{\beta-z}{1-\bar{\beta} z}
$$

is a self-inverse automorphism of $U$. Thus for arbitrary $f \in H^{2}$,

$$
\left\|C_{\varphi} f\right\|=\left\|\left(C_{\varphi} C_{\nu}\right) C_{\nu} f\right\|=\left\|C_{\nu} f\right\|,
$$

where we have used the fact that $C_{\varphi} C_{\nu}=C_{\nu \circ \varphi}$ is an isometry (since $\nu \circ \varphi$ is inner and fixes 0 ). Thus

$$
\sup _{\alpha \in U}\left\|C_{\varphi}\left(\frac{k_{\alpha}}{\left\|k_{\alpha}\right\|}\right)\right\|=\sup _{\alpha \in U}\left\|C_{\nu}\left(\frac{k_{\alpha}}{\left\|k_{\alpha}\right\|}\right)\right\| .
$$

Employing Lemma 4.3, we have

$$
\begin{aligned}
\left\|C_{\nu}\left(\frac{k_{\alpha}}{\left\|k_{\alpha}\right\|}\right)\right\|^{2} & =\left(1-|\alpha|^{2}\right) \frac{|\beta|^{2}+1+2 \operatorname{Re} \frac{\beta(\bar{\alpha}-\bar{\beta})}{1-\bar{\alpha} \beta}}{|1-\bar{\alpha} \beta|^{2}-|\bar{\alpha}-\bar{\beta}|^{2}} \\
& =\frac{|\beta|^{2}+1+2 \operatorname{Re} \frac{\beta(\bar{\alpha}-\bar{\beta})}{1-\bar{\alpha} \beta}}{1-|\beta|^{2}} .
\end{aligned}
$$


As $\alpha \rightarrow \beta /|\beta|$ from within $U$, the last quantity approaches $(1+|\beta|) /(1-|\beta|)$, which is the square of the norm of $C_{\varphi}$ by Theorem 4.2.

Remark. Because the normalized kernels go to 0 weakly in $H^{2}$ as $|\alpha| \rightarrow 1^{-}$, the proof of Theorem 4.2 shows that the essential norm of an innerfunction-induced composition operator equals its norm. This fact was first proved by Shapiro [1987, Theorem 2.5].

Given Theorem 4.2, the issue of whether reproducing kernels determine composition-operator norms is unresolved.

We now present an example that remedies both defects of the map $\psi$ above, namely, a univalent map $\gamma$ of the disk into itself such that $\left\|C_{\gamma}\right\|$ is not determined by the action of $C_{\gamma}$ on the set of normalized reproducing kernels.

Theorem 4.4. Suppose $\gamma(z)=2 /(3-z)$, and set

$$
S:=\sup _{\alpha \in U}\left\|C_{\gamma}\left(\frac{k_{\alpha}}{\left\|k_{\alpha}\right\|}\right)\right\|, \quad S^{*}:=\sup _{\alpha \in U}\left\|C_{\gamma}^{*}\left(\frac{k_{\alpha}}{\left\|k_{\alpha}\right\|}\right)\right\| .
$$

Then $\left\|C_{\gamma}\right\|>S>S^{*}$.

Maple plots over the unit disk of the functions $\alpha \mapsto$ $\left\|C_{\gamma}\left(k_{\alpha} /\left\|k_{\alpha}\right\|\right)\right\|$ and $\alpha \mapsto\left\|C_{\gamma}^{*}\left(k_{\alpha} /\left\|k_{\alpha}\right\|\right)\right\|$ suggest that each of these functions attains a maximum along the positive real axis. Thus Figure 2 provides numerical evidence supporting the validity of the second inequality of Theorem 4.4.

Proof of Theorem 4.4. We show explicitly that

$$
S=\sqrt{\frac{(25-2 \sqrt{5})(-2+\sqrt{125})}{40+21 \sqrt{5}}} \approx \sqrt{2.167}
$$

To prove (4.3), use the formula of Lemma 4.3 (with $\left.\alpha=r e^{i \theta}\right)$ to obtain the value

$$
\frac{\left(1-r^{2}\right)\left(72-108 r \cos \theta+40 r^{2}\right)}{\left(8-12 r \cos \theta+4 r^{2}\right)\left(9-12 r \cos \theta+4 r^{2}\right)}
$$

for $\left\|C_{\gamma}\left(k_{\alpha} /\left\|k_{\alpha}\right\|\right)\right\|^{2}$. Replacing $\cos \theta$ by $y$, differentiating with respect to $y$, and simplifying gives

$\frac{3 r\left(1-r^{2}\right)\left(144+161 r^{2}+44 r^{4}-432 r y-240 r^{3} y+324 r^{2} y^{2}\right)}{\left(-2-r^{2}+3 r y\right)^{2}\left(-9-4 r^{2}+12 r y\right)^{2}}$.

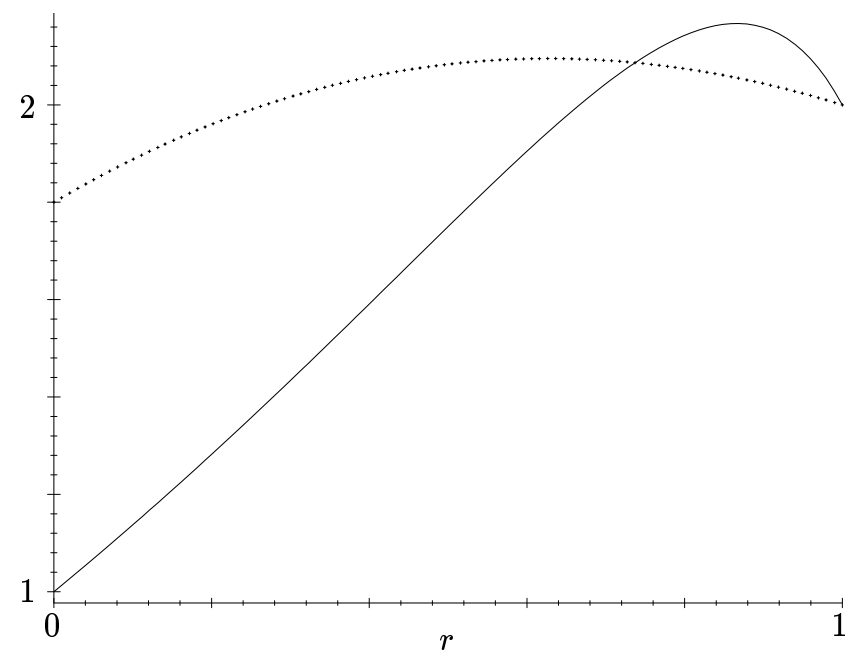

FIGURE 2. Graphs of $\left\|C_{\gamma}\left(k_{r} /\left\|k_{r}\right\|\right)\right\|^{2}$ (solid) and $\left\|C_{\gamma}^{*}\left(k_{r} /\left\|k_{r}\right\|\right)\right\|^{2}$, for $0 \leq r<1$.

The discriminant of the quadratic expression in $y$ in parentheses in the numerator is negative for each $r \in[0,1)$, and since the expression is positive when $y=0$, we have an increasing function in $y$. Thus for fixed $r$ the quantity on the right of (4.4) is maximized when $\theta=0$, or $y=1$. We now know that

$$
S^{2}=\sup _{r \in[0,1)} \frac{\left(1-r^{2}\right)\left(72-108 r+40 r^{2}\right)}{\left(8-12 r+4 r^{2}\right)\left(9-12 r+4 r^{2}\right)} .
$$

Calculus shows that the supremum is attained at $r_{S}=\frac{14}{11}-\frac{2}{11} \sqrt{5}$, which gives (4.3) upon substitution.

The value of $S_{*}$ is even easier to compute. We have

$$
\left\|C_{\gamma}^{*}\left(k_{\alpha} /\left\|k_{\alpha}\right\|\right)\right\|^{2}=\frac{1-r^{2}}{1-\left|2 /\left(3-r e^{i \theta}\right)\right|^{2}} .
$$

Here, it's clear that for fixed $r$ the quantity on the right of the preceding equation is maximized when $\theta=0$. Thus

$$
S_{*}^{2}=\sup _{r \in[0,1)} \frac{1-r^{2}}{1-(2 /(3-r))^{2}},
$$

and calculus yields

$$
S_{*}=\sqrt{\frac{(\sqrt{33}-1)^{2}(9-\sqrt{33})}{4(3+\sqrt{33})}} \approx \sqrt{2.095} .
$$


To complete the proof of Theorem 4.4, we must show that the norm of $C_{\gamma}$ exceeds $S$. What we need is a norm-one $H^{2}$ function $f$ such that $\left\|C_{\gamma} f\right\|>S$ or $\left\|C_{\gamma}^{*} f\right\|>S$.

We first experimented with functions of the form

$$
f(z)=\frac{1 /(1-z)^{\beta}}{\left\|1 /(1-z)^{\beta}\right\|},
$$

where $0<\beta<\frac{1}{2}$, but numerical computations were inconclusive. We then considered the family

$$
f_{\lambda}(z)=\frac{\left(k_{r_{S}}\right)^{\lambda}}{\left\|\left(k_{r_{S}}\right)^{\lambda}\right\|}
$$

for $\lambda>0$, where $r_{S}$ is the number yielding the supremum in (4.5). The reason for considering this family is clear: when we maximize $\left\|C_{\gamma} f_{\lambda}\right\|$ over $\lambda$ we must get at least $S$ since $\left\|C_{\gamma} f_{1}\right\|=S$. We computed $\left\|C_{\gamma} f_{\lambda}\right\|$ numerically using the integral representation of the $H^{2}$ norm (the integrals being evaluated with Simpson's rule). We remark that these norm computations using the integral representation stabilized fairly quickly with increasing numbers of partition points; hence, the integral normrepresentation appears to be a viable experimental tool. Results were encouraging; in particular, the plot shown in Figure 3 suggests that $\left\|C_{\gamma} f_{\lambda}\right\|$ exceeds $S$ for some values of $\lambda$.

If one can prove Figure 3 is accurate, all issues are settled. To test the accuracy of the plot we symbolically computed the derivative of $\left\|C_{\gamma}\left(f_{\lambda}\right)\right\|^{2}$ with respect to $\lambda$ (differentiating under the integral sign) and then numerically computed the value of the derivative at 1 . The result was negativecomforting, but task of verifying that the negative result was not due to numerical error appeared daunting (to the authors, at least). We abandoned this family of test functions.

The family of test functions that settled the issue for us is

$$
f_{r, s}=\frac{k_{r}+k_{s}}{\left\|k_{r}+k_{s}\right\|}
$$

for $0 \leq r, s<1$. Since the set of all linear combinations of reproducing kernels is dense in $H^{2}$, some

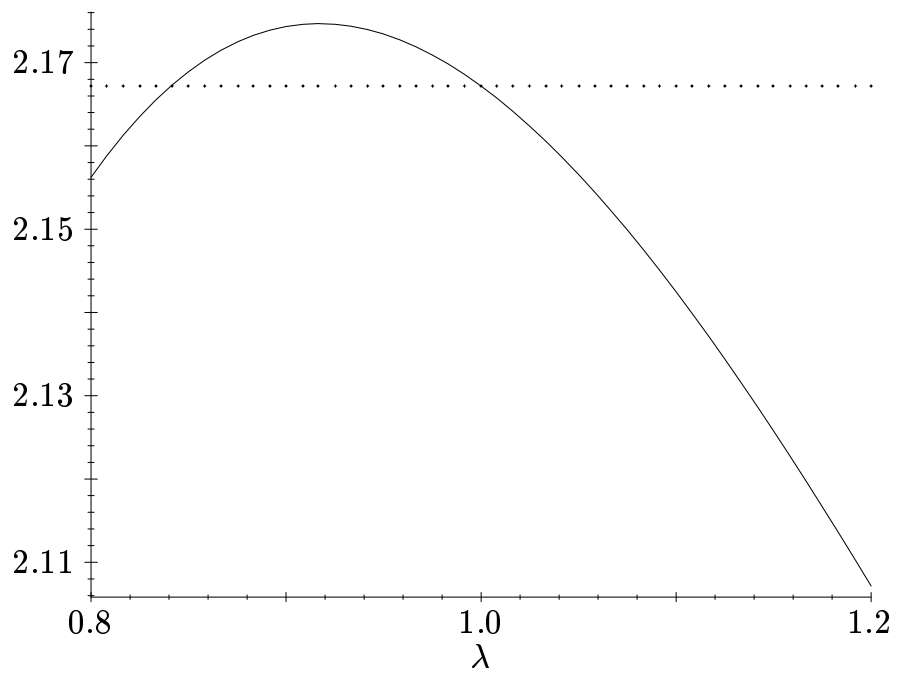

FIGURE 3. Graph of $\left\|C_{\gamma} f_{\lambda}\right\|^{2}$ for $0.8 \leq \lambda \leq 1.2$. The dotted line is at height $S^{2}$.

linear combination of reproducing kernels (normalized to have length one) must under the action of $C_{\gamma}$ yield an image with norm exceeding $S$ (provided the numerical results depicted in Figure 3 are accurate). Figure 4 is a plot suggesting that the family $f_{r, s}$ does the trick.

Fortunately, $\left\|C_{\gamma}^{*} f_{r, s}\right\|^{2}$ may be computed exactly. In the following, we set $r=0$ and $s=\frac{17}{20}$, so that

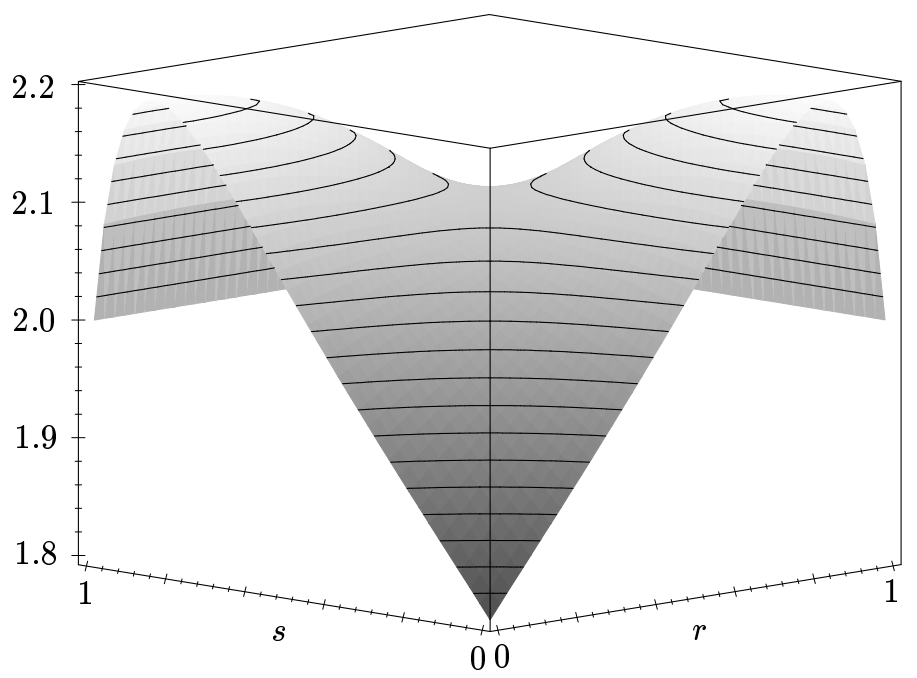

FIGURE 4. Graph of $\left\|C_{\gamma}^{*} f_{r, s}\right\|^{2}$, for $0 \leq r, s<1$. 
$f=\left(1+k_{17 / 20}\right) /\left\|1+k_{17 / 20}\right\|$. Then, as we wished to show,

$$
\begin{aligned}
\left\|C_{\gamma}^{*} f\right\|^{2} & =\frac{\left\|C_{\gamma}^{*}\left(1+k_{s}\right)\right\|^{2}}{3+1 /\left(1-s^{2}\right)} \\
& =\frac{111}{733}\left\langle k_{\gamma(0)}+k_{\gamma(s)}, k_{\gamma(0)}+k_{\gamma(s)}\right\rangle \\
& =\frac{32708888}{1490555} \approx 2.194>S^{2} .
\end{aligned}
$$

We conclude with a question.

Question 4.5. What is the norm of $C_{\gamma}$ ?

\section{REFERENCES}

[Cowen 1988] C. C. Cowen, "Linear fractional composition operators", Integral Equations and Operator Theory 11 (1988), 151-160.
[Cowen and MacCluer 1995] C. C. Cowen and B. D. MacCluer, Composition Operators on Spaces of Analytic Functions, CRC Press, Boca Raton, FL, 1995.

[Duren 1970] P. L. Duren, Theory of $H^{p}$ spaces, Academic Press, New York, 1970.

[Littlewood 1925] J. E. Littlewood, "On inequalities in the theory of functions", Proc. London Math. Soc.(2) 23 (1925), 481-519.

[Nordgren 1968] E. A. Nordgren, "Composition Operators", Canadian J. Math. 20 (1968), 442-449.

[Ryff 1966] J. V. Ryff, "Subordinate $H^{p}$ functions", Duke Math. J. 33 (1966), 347-354.

[Shapiro 1987] J. H. Shapiro, "The essential norm of a composition operator", Ann. Math. 125 (1987), 375404 .

Paul S. Bourdon, Department of Mathematics, Washington and Lee University, Lexington, VA 24450 (pbourdon@wlu.edu)

Received July 12, 1995; accepted April 12, 1996 